\title{
Pacemaker channel dysfunction in a patient with sinus node disease
}

\author{
Eric Schulze-Bahr, ${ }^{1,2}$ Axel Neu, ${ }^{3}$ Patrick Friederich, ${ }^{4}$ U. Benjamin Kaupp ${ }^{5}$ \\ Günter Breithardt, ${ }^{1,2}$ Olaf Pongs, ${ }^{3}$ and Dirk Isbrandt ${ }^{3}$
}

\author{
${ }^{1}$ Genetics of Arrhythmias, Molecular Cardiology Section, Institute for Arteriosclerosis Research, University of Münster, \\ Münster, Germany \\ ${ }^{2}$ Department of Cardiology and Angiology, Hospital of the University of Münster, Münster, Germany \\ ${ }^{3}$ Institute for Neural Signal Transduction, Center for Neurobiology Hamburg (ZMNH), University of Hamburg, \\ Hamburg, Germany \\ ${ }^{4}$ Department of Anesthesiology, University Hospital Hamburg, Hamburg, Germany \\ ${ }^{5}$ Institute for Biological Information Processing, Research Center Jülich, Jülich, Germany
}

The cardiac pacemaker current $I_{f}$ is a major determinant of diastolic depolarization in sinus nodal cells and has a key role in heartbeat generation. Therefore, we hypothesized that some forms of "idiopathic" sinus node dysfunction (SND) are related to inherited dysfunctions of cardiac pacemaker ion channels. In a candidate gene approach, a heterozygous 1-bp deletion (1631delC) in exon 5 of the human HCN4 gene was detected in a patient with idiopathic SND. The mutant HCN4 protein (HCN4-573X) had a truncated C-terminus and lacked the cyclic nucleotide-binding domain. COS-7 cells transiently transfected with HCN4-573X cDNA indicated normal intracellular trafficking and membrane integration of HCN4-573X subunits. Patch-clamp experiments showed that HCN4-573X channels mediated $I_{f}$-like currents that were insensitive to increased cellular cAMP levels. Coexpression experiments showed a dominant-negative effect of HCN4-573X subunits on wild-type subunits. These data indicate that the cardiac $I_{f}$ channels are functionally expressed but with altered biophysical properties. Taken together, the clinical, genetic, and in vitro data provide a likely explanation for the patient's sinus bradycardia and the chronotropic incompetence.

This article was published online in advance of the print edition. The date of publication is available

from the JCI website, http://www.jci.org. J. Clin. Invest. 111:1537-1545 (2003). doi:10.1172/JCI200316387.

\section{Introduction}

Sinus node dysfunction (SND) is the major cause necessitating pacemaker implantation and accounts for approximately half of all patients requiring a pacemaker (1). The disease commonly occurs in adults with acquired heart disease, during antiarrhythmic therapy, or after surgically corrected, congenital heart disease. In a significant portion of patients, however, SND appears in the absence of identifiable cardiac abnormalities or other associated conditions ("idiopathic" SND) (2). A familial occurrence of idiopathic SND has been reported in

Received for publication July 10, 2002, and accepted in revised form February 18, 2003.

Address correspondence to: Eric Schulze-Bahr, AG Genetics of Arrhythmias, Molekular-Kardiologie, Institut für Arterioskleroseforschung an der Westfälischen WilhelmsUniversität Münster, Domagkstrasse 3, D-48149 Münster, Germany. Phone: 49-251-83-52982; Fax: 49-251-83-52980; E-mail: heart@uni-muenster.de.

Conflict of interest: The authors have declared that no conflict of interest exists.

Nonstandard abbreviations used: sinus node dysfunction (SND); sinoatrial node (SAN); cyclic nucleotide-binding domain (cNBD); hyperpolarization-activated cyclic nucleotide-gated channels (HCN); beats per minute (bpm); dual-chamber (DDD); electrocardiography (ECG); $\mathrm{CMV}$ promoter $\left(\mathrm{P}_{\mathrm{CMV}}\right)$; human elongation factor $1 \alpha$ promoter $\left(\mathrm{P}_{\mathrm{EF}-1 \alpha}\right)$; enhanced green fluorescent protein (EGFP); internal ribosomal entry site (IRES); midpoint potential $\left(\mathrm{V}_{1 / 2}\right)$. some instances (2-7). These findings and observations from twin studies indicate a role for genetic factors in SND and heart rate control $(8,9)$.

The sinoatrial node (SAN) is the physiological pacemaker in the human heart and is predominantly responsible for autonomous heart beat generation. Heart rate control is achieved through control of SAN pacemaking by the autonomic nervous system; cholinergic or $\beta$-adrenergic stimulation either slows or accelerates the spontaneous SAN activity (10). Pacemaking activity depends on a phase of spontaneous, slow membrane depolarization that occurs between cardiac action potentials (i.e., during diastole). Several ionic currents apparently contribute to the time course and slope of diastolic membrane depolarizations (e.g., the T-type and L-type $\mathrm{Ca}^{2+}$ currents), some of which are directly regulated by the autonomic nervous system (10). In particular, the activity of the hyperpolarization-activated nonselective cationic current $I_{f}$ is cAMP regulated (11). This involves a direct interaction of cAMP with the cyclic nucleotide-binding domain (cNBD) of $I_{f}$ channels (11, 12) and elicits a positive shift in the voltage dependence of $I_{f}$ channel activation accompanied by an increase in current amplitude. Eventually, this leads to accelerated diastolic depolarization and electrical impulse generation in SAN. Conversely, a decrease in cellular cAMP levels (e.g., during muscarinic stimulation) leads to a decrease in $I_{f}$ channel activity and thereby slows the 
heart rate. Apparently, cAMP sensitivity of pacemaker channels represents a key element in the response of heart rate to sympathetic stimulation $(13,14)$.

Hyperpolarization-activated cyclic nucleotide-gated (HCN) subunits are the molecular components of $I_{f}$ channels. Two of the four known genes encoding HCN channel subunits (15-19), HCN2 and HCN4, are predominantly expressed in the heart (20). Their in vitro properties closely resemble those of native $I_{f}$ channels (15-18, 21-23). Therefore, $H C N$ genes have been suggested as candidates underlying disorders of heartbeat generation $(13,17)$.

In a previous study, we excluded the presence of HCN2 mutations in a series of ten patients with SND (24). Here, we report on the investigation of the HCN4 gene in the same population with idiopathic SND. In one index patient, a heterozygous 1-bp deletion mutation was detected that gave rise to a C-terminally truncated $\mathrm{HCN} 4$ protein (HCN4-573X) lacking the C-terminal cNBD domain. In vitro-expressed HCN4-573X channels mediated $I_{f}$-like currents comparable to those of wild-type HCN4 but were unable to respond to increases in intracellular cAMP levels. In coexpression experiments with wild-type subunits, current properties of HCN4-573X subunits were predominant. It is likely that altered $I_{f}$ channel properties in the index patient are associated with the observed clinical SND phenotype. Our investigation supports the hypothesis that idiopathic SND is in part genetically determined and may be linked to mutations in a cardiac pacemaker channel gene.

\section{Methods}

\section{Clinical records}

A 66-year-old woman was admitted to a community hospital with a fractured nasal bone after a severe syncope. On admission, the patient had marked sinus bradycardia (41 beats per minute [bpm]) (Figure 1a) and intermittent episodes of atrial fibrillation (Figure 1b). The plasma electrolytes were normal and the patient was not taking any medication. The patient's physician reported that bradyarrhythmia and intermittent atrial fibrillation had been known and tolerated for over 20 years. The results of a complete invasive cardiological examination (right and left ventriculography, coronary angiography, and programmed ventricular stimulation) were normal. In the absence of detectable heart disease, the diagnosis of idiopathic SND was made and a dual-chamber (DDD) pacemaker was implanted. During routine pacemaker followup, the patient's physical performance improved markedly, and there was no recurrence of syncope. An evaluation of the patient's P-waves (as obtained from the P-wave histogram during pacemaker interrogation) showed that the majority (>98\%) of the atrial rates were below $60 \mathrm{bpm}$ within the first 68 days after pacemaker implantation (Figure 1c). Episodes of fast atrial rates (i.e., atrial tachyarrhythmias), either isolated or together with a slow ventricular rate response (suggesting the presence of

high-grade atrioventricular conduction block), were not observed during pacemaker interrogation. During exercise electrocardiography (ECG), the patient had sinus rhythm, and the maximal heart rate was $101 \mathrm{bpm}$ at a maximal work load of $150 \mathrm{~W}$. This was markedly below the predicted maximal heart rate when adapted for age and gender (150-184 bpm) (Figure 1d) (25).

The family history of the female index patient was unremarkable; three of her four children (between 32 and 39 years of age) that were available for clinical and genetic investigations had normal 12-lead ECGs with sinus rhythms (65, 75, and $65 \mathrm{bpm}$, respectively) and exercise ECGs with regular chronotropic responses.

In addition to this family, nine other unrelated index families with idiopathic SND were available for genetic investigations; three had familial sinus bradycardia (without atrial fibrillation), two had "lone atrial fibrillation," three had "sporadic" SND, and one previously reported large family had autosomal dominant sinoatrial disease (24).

Genomic structure, chromosomal localization, and mutation detection of the $\mathrm{HCN} 4$ gene

A BLAST search of the National Center for Biotechnology Information GenBank high-throughput genomic sequences database using the HCN4 cDNA (GenBank accession number AJ238850) as a probe identified a

a

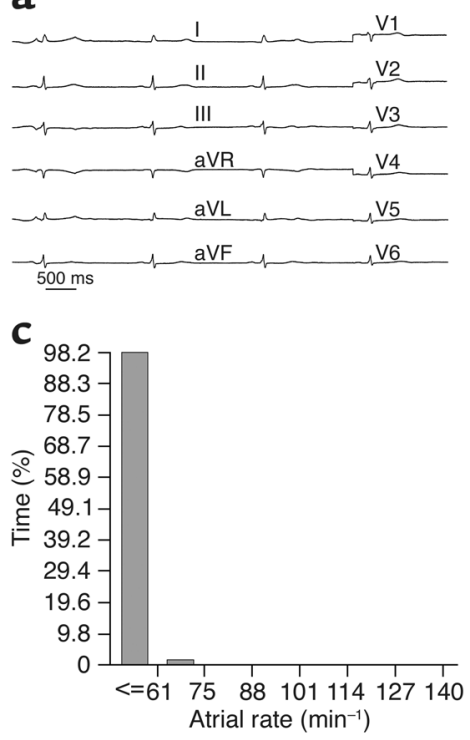

Figure 1

(a) Baseline electrocardiogram of the index patient showing marked sinus bradycardia (41 bpm). (b) ECG recorded during an episode of intermittent atrial fibrillation. (c) P-wave histograms obtained from pacemaker interrogation after implantation. At day $68,98 \%$ of the atrial rates were below a threshold level of $60 \mathrm{bpm}$. (d) Heart rates (at sinus rhythm) of the index patient during bicycle exercise, before pacemaker implantation. The vertical dotted line and gray area indicate the predicted mean maximal work performance \pm SD (with respect to the patient's age [65-70 years] and gender) (25). The dotted horizontal line indicates the predicted heart rate value during maximal work load for women 66 years of age (25). 
Table 1

Genomic primers $\left(5^{\prime}-3^{\prime}\right)$ used for complete exon amplification of the HCN4 gene

\begin{tabular}{|c|c|c|}
\hline Exon & \multicolumn{2}{|c|}{ Primer } \\
\hline & Sense & Antisense \\
\hline $1-1$ & GCGGCGCCGCGCTCCTGCCC & CGCTCGGGCTCGGCCGCCAG \\
\hline $1-2$ & CGGCAGCAGTCACGGACACCTGC & GGAAAGTTAACTCCGGCTGGGAGGC \\
\hline 2 & ТСТСТСТTССTGGСGACTGACCC & GGTCAAGAACTTACTAGTATTTGTCC \\
\hline 3 & GAGCAGTGCCCACCAGCAGCTC & GCCACCСТACСTCTGGAGAGC \\
\hline 4 & AGGTTGAGGTGAGTAGGTGGCAGG & СTGAAACTCAGATTCTCATCTCAGAGG \\
\hline 5 & AGATCTCAAGGAACCAAGTTTAGCC & AGGGTGGATTGGGACACGGGAAGG \\
\hline 6 & ССТTCССGTGTСССААТССАСССТ & СССТACССТGGGCTCACAGACACC \\
\hline 7 & CCATTTGGTGGGGAAGAGGCATCC & ATCAGGTGCAGACCTGGCTTAGGC \\
\hline $8-1$ & СССGСТСTGСССТGAGTGССТGT & CCGAGGTTGCCCAGCCCAGATCC \\
\hline $8-2$ & ACTTCTGTGGCCATAGCCСTCAC & GTCGGAGGAGGACAGGGAGCCACC \\
\hline $8-3$ & TCCCACACCATCAGCTGGCGTAGC & TGCCACAAGGGACGGCGGCTCAGG \\
\hline $8-4$ & CGGGGAGTTGTCCСTAGGTCTGG & CTGGGGAAGAGCGGGAAGGCAGC \\
\hline $8-5$ & СTCAGGACGGGGCGCAGACTCTC & AGAGAAAAGAAGAAAGAAGAGGGAA \\
\hline
\end{tabular}

sequence harboring HCN4 gene exons 1-8 (Figure 2; accession number AC009660). The Genebridge 4 wholegenome radiation hybrid panel (Invitrogen, Karlsruhe, Germany) was screened by PCR using HCN4-specific primers. PCR reactions were performed with $25 \mathrm{ng}$ of DNA for all 93 hybrid clones and with human and hamster genomic DNA controls. Two independent PCR reactions amplifying genomic fragments of exon 1/intron 1 or exon 8 , respectively, were used to analyze the chromosomal localization of the HCN4 gene. The primers were derived from genomic sequences of the HCN4 gene identified as described above. Conditions were as follows: for PCR A (primer AAAGCCGTGGAGCGCGAACAGGAG [sense] and primer CCCAGCGCAAGGCAGGAAAGTTA [antisense] $), 5$ minutes at $95^{\circ} \mathrm{C}(1$ cycle $)$ and 30 seconds

\section{Figure 2}

Mutation detection in the HCN4 gene. (a) The human HCN4 gene consists of eight exons. The minimal exon size was 141 bp (exon 5), and the maximal size was 1,465 bp (exon 8); the largest intron was intron $1(\sim 24 \mathrm{~kb})$ and the smallest intron 5 (102 bp). The functional domains of the wild-type HCN4 channel are delineated below. P, pore region; 1-6, transmembrane domains. (b) Electropherogram after direct sequencing of an index patient with SND. A heterozygous 1-bp deletion (1631delC) in HCN4 resulted in a superimposing sequence pattern consisting of the wild-type and mutant exon 5 sequence. (c) The heterozygous deletion mutation induces an Ecil restriction site in exon 5; after restriction enzyme analysis, the uncut wild-type fragment (380 bp) and two Ecil fragments (200 and 180 bp, cut mutant fragment) were found in the index patient. Her three healthy children had the wild-type configuration. The left lane shows the size standard in base pairs (pUC19 DNA/Mspl). (d) Schematic topology of HCN4 channels with six transmembrane segments (S1-S6) and intracellular $\mathrm{N}$ - and $\mathrm{C}$-termini. Because of the reading frame shift in the nucleotide sequence, a resulting premature stop codon deleted the C-terminally located cNBD in HCN4-573X that is replaced by 29 novel amino acids (thick gray line). The relative sizes of the transmembrane segments and $\mathrm{N}$ - or C-termini are not drawn to scale. at $95^{\circ} \mathrm{C}, 30$ seconds at $65^{\circ} \mathrm{C}$, and 45 seconds at $72^{\circ} \mathrm{C}(30$ cycles $)$ in buffer containing $5 \%$ DMSO and Taq polymerase (Invitrogen); and for PCR B (primer TGCCACCCCCTCTGTCTTTGTTTG [sense] and primer CCTСССТСССССТСССТСССТСТА [antisense]), 5 minutes at $95^{\circ} \mathrm{C}(1$ cycle $)$ and 30 seconds at $95^{\circ} \mathrm{C}, 30$ seconds at $62^{\circ} \mathrm{C}$, and 30 seconds at $72^{\circ} \mathrm{C}(30$ cycles) in buffer containing $5 \%$ DMSO and Taq polymerase (Invitrogen). PCR products were separated by agarose gel electrophoresis. PCR results were submitted to the Radiation Hybrid Mapping Server at the Whitehead Institute (http://wwwgenome.wi.mit.edu/cgi-bin/contig/rhmapper). A cutoff of 15 was chosen for the LOD score.

For mutation screening, intronic primers were developed (Table 1) that encompassed the complete coding sequence, the splice sites, and the adjacent lariat sites. Because of the large size or a high CG content, exons 1 and 8 were subdivided into 2 and 5 amplicons, respectively. Genomic DNA was extracted from peripheral lymphocytes of the patients, family members, and control individuals using standard techniques (26). A total of 181 unrelated and healthy individuals (26) served as controls for verification of sequence variations from the published HCN4 cDNA sequence. Sequencing of PCR products and separation of sequencing products were performed using the BigDye RR Terminator AmpliTaq-Kit version 3.0 and the ABI3700 system (PerkinElmer Applied-Biosystems, Weiterstadt, Germany).

a

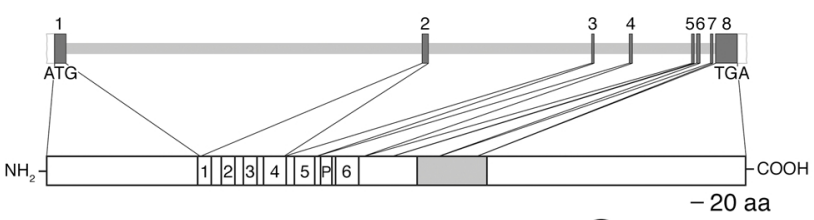

b

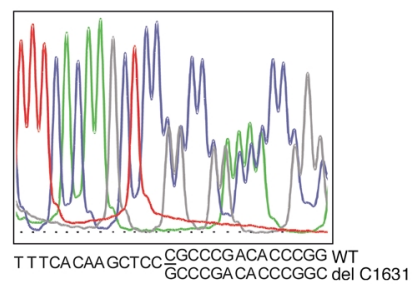

d

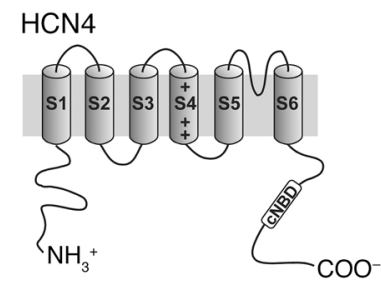

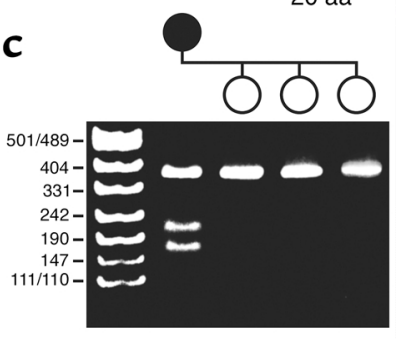

HCN4-573X

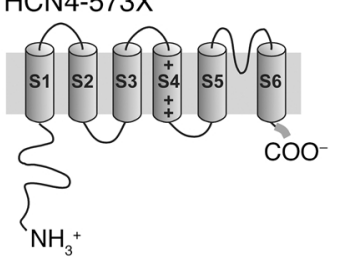



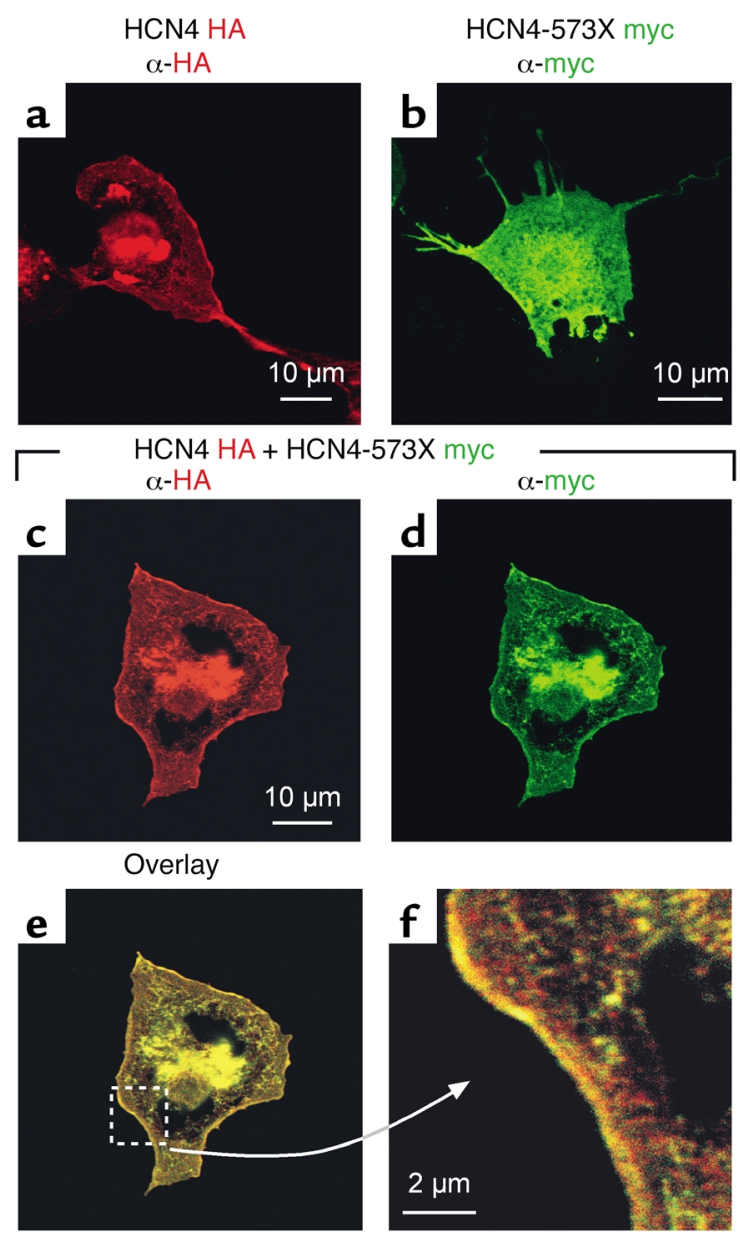

Electropherograms were compared with the $\mathrm{HCN} 4$ wild-type sequence (GenBank accession number AJ238850) using the SeqMan program 5.0 (DNASTAR Inc., Madison, Wisconsin, USA).

Generation of wild-type and mutant HCN4 expression constructs

Immunocytochemistry. Full-length wild-type $\mathrm{HCN} 4 \mathrm{cDNA}$ was cloned into the vector pcDNA1Amp (Invitrogen), and the identified mutation was introduced by sitedirected mutagenesis. Subsequently, $\mathrm{N}$-terminal epitope

\section{Figure 3}

Immunostaining of HCN4 and HCN4-573X in COS-7 cells. Wild-type and HCN4-573X channels were transiently expressed in COS-7 cells. For detection, wild-type HCN4 was HA-tagged and HCN4-573X was myc-tagged. Both channels showed comparable immunostains (a and $\mathbf{b}$ ) with immunoreactivity detectable in perinuclear regions and at the plasma membrane. When coexpressed (c-f), the immunostaining patterns did not change and completely overlapped, and they were particularly concentrated in the cell membrane $(\mathbf{f})$.

sequences (wild-type, HA epitope; mutant, myc epitope) were added to the cDNAs. The pcDNA1Amp-HCN4 vector $(1 \mu \mathrm{g})$ was used for transient expression of either tagged wild-type or mutant channels or cotransfection of both constructs (Figure 3 ) in COS-7 cells (American Type Culture Collection, Rockville, Maryland, USA).

Electrophysiological experiments. For transient expression and electrophysiological characterization of either wildtype or mutant channels in COS-7 cells (Figures 4 and 5), full-length wild-type or mutant $\mathrm{HCN} 4 \mathrm{cDNA}$ was cloned into the first polylinker of the expression vector $\mathrm{pBud}$ CE4.1 (Invitrogen). Expression was therefore driven by the $\mathrm{CMV}$ promoter $\left(\mathrm{P}_{\mathrm{CMV}}\right)$. In order to enable selection of transfected cells, a cDNA encoding EGFP (enhanced green fluorescent protein; Clontech, Palo Alto, California, USA) was inserted into the second polylinker of pBud-CE4.1 that already contained untagged full-length (wild-type or mutant) HCN4 cDNA in the first polylinker. For coexpression experiments (Figure 6), another expression plasmid ( $\mathrm{pCo}-\mathrm{HCN} 4)$ was constructed that contained the mutant HCN4-573X cDNA in the first polylinker of $\mathrm{pBud}-\mathrm{CE} 4.1$ (under control of $\mathrm{P}_{\mathrm{CMV}}$ ) and the wild-type $\mathrm{HCN} 4 \mathrm{cDNA}$ in the second polylinker (under control of human elongation factor $1 \alpha$ promoter ( $\left.\mathrm{P}_{\mathrm{EF}-1 \alpha}\right)$; Figure 6a). In addition, an internal ribosomal entry site (IRES) EGFP element was cloned $3^{\prime}$ to wildtype HCN4 to allow identification of transfected cells. Hence, EGFP expression was coupled to the expression of wild-type HCN4, since both open reading frames were located on one transcript.

$R T-P C R$. The relative amounts of wild-type and mutant HCN 4 transcripts in coexpression experiments (Figure 6b) were determined as follows: total RNA from $10^{6}$ cells transiently transfected with either pCo-HCN4,

Table 2

Exon/intron boundaries of the HCN4 gene

\begin{tabular}{|c|c|c|c|c|c|}
\hline Exon & cDNA nucleotides & Amino acid & Splice donor site & Splice acceptor site & Intron size(bp) \\
\hline 1 & $1-785$ & $1-262$ & ...ccctggcgccATGGACAAGC... & ...GTGACTTCAGgtaaggggcc... & $23.7 \mathrm{~kb}$ \\
\hline 2 & $786-1209$ & $263-403$ & ...ctctgggcagATTTTACTGG... & ...GTGGGAAGAGgtaagtggtc... & $11.5 \mathrm{~kb}$ \\
\hline 3 & $1210-1371$ & $404-457$ & ...ctgctggcagATCTTCCACA... & ...CAACATGGTGgtgagtgcgc... & $2.3 \mathrm{~kb}$ \\
\hline 4 & $1372-1590$ & $458-530$ & ...ctgtccacagAACAACTCCT... & ...CCAGGAAAAGgtaggtctac... & $4.3 \mathrm{~kb}$ \\
\hline 5 & $1591-1732$ & $531-579$ & ...ccggcctcagTACAAGCAGG... & ...CCTGCGGGAGgtgagctgtg... & 102 bp \\
\hline 6 & $1733-1978$ & $580-659$ & ...tgtccggcagGAGATCATCA... & ...TACTTTGGAGgtgaggcagc... & 701 bp \\
\hline 7 & 1979-2143 & $660-714$ & ...gctctggcagAGATCTGCCT. & ...GACCGCATTGgtgaggggga... & $139 \mathrm{bp}$ \\
\hline 8 & 2144-3609 & $715-1203$ & ...tgtggeccagGCAAGAAGAA... & ...CAATCTATGAgctgggccct... & 3'-UTR \\
\hline
\end{tabular}

The gene contains eight exons; the cDNA is composed of 3,609 nucleotides resulting in 1,203 amino acids. The intronic splice sites adhere to the "AG-GT" rule; these nucleotides are underlined. Coding nucleotides are marked in capital letters. UTR, untranslated region. 


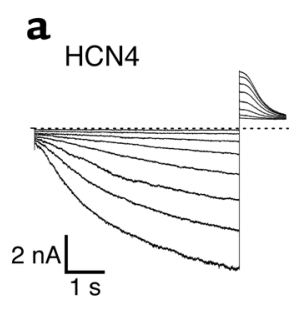

b
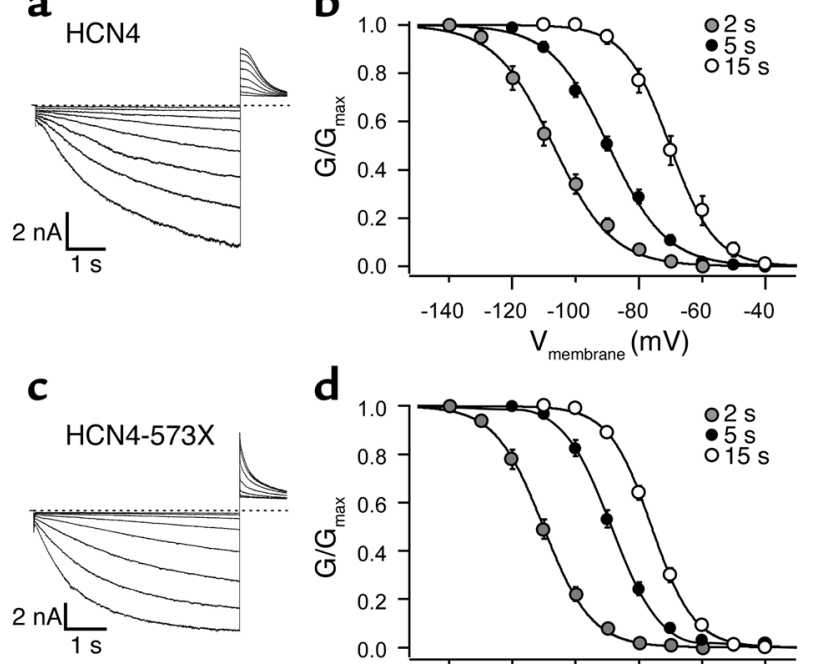

d

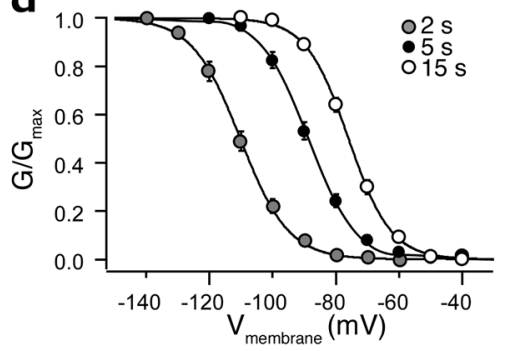

Figure 4

Functional characterization of homomeric HCN4 and HCN4-573X channels in COS-7 cells under control conditions. Representative current traces of wild-type (a) or HCN4-573X (c) channels elicited by 5-second-long hyperpolarizing voltage steps from $-40 \mathrm{mV}$ to $-120 \mathrm{mV}$ under control conditions. Relative open probability of wild-type (b) and HCN4-573X (d) channels was dependent on test-pulse duration (gray circles, 2 seconds; black circles, 5 seconds; white circles, 15 seconds). Absence of error bars indicates errors smaller than the symbol size.

pcDNA1Amp-HCN4, or pcDNA1Amp-HCN4-573X was isolated (Purescript RNA isolation Kit, Biozym, Oldendorf, Germany) and reverse-transcribed (Superscript II, Invitrogen). HCN4 cDNA fragments were amplified by PCR using $1 \mu \mathrm{l}$ of reverse transcription reaction (forward primer, 5'-GGCATGTCCGACGTCTGGCTCAC-3'; reverse primer, $5^{\prime}$-TCACGAAGTT GGGGTCCGC ATTGG-3'). To differentiate between wild-type and mutant PCR fragments, we digested the fragments with EciI (MBI Fermentas, St. Leon-Roth, Germany) (see Figure 2c) and separated them by agarose gel electrophoresis. A CCD camera-acquired image (Biometra, Göttingen, Germany) of the ethidium bromide-stained gel was analyzed using AIDA image analysis software (Raytest, Sprockhöfel, Germany).

Cell culture, transfection, and immunostaining

For immunocytochemical localization, COS-7 cells were transiently transfected with wild-type and/or mutant cDNAs. COS-7 cells were seeded on coverslips in $35-\mathrm{mm}$ dishes 1 day before transfection, which was performed using $3.5 \mu \mathrm{l}$ of lipofectamine reagent (Invitrogen) according to the manufacturer's protocol and $1.5 \mu \mathrm{g}$ of the respective plasmid DNA. Two days after transfection, cells were fixed on coverslips with $2 \%$ paraformaldehyde. After a blocking incubation $\left(1\right.$ hour at $37^{\circ} \mathrm{C}$ with $3 \%$ goat serum and $5 \%$ bovine serum albumin [SigmaAldrich, Deisenhoffen, Germany] in phosphate-buffered saline), immunostaining was performed with the affinity-purified anti-myc monoclonal antibody (dilution,
1:100; Roche Diagnostics, Mannheim, Germany) and the polyclonal anti-HA tag antibody (dilution, 1:500; Babco, Richmond, California, USA). Alexa546-labeled anti-rabbit IgG or Alexa488-labeled anti-mouse IgG (dilution, 1:2000; Molecular Probes, Eugene, Oregon, USA) served as secondary immunofluorescent reagents. Confocal images were obtained in sequential scan mode using a Leica TCS NT laser scanning microscope (Leica, Bensheim, Germany).

Electrophysiological recordings

Transfection of COS-7 cells for electrophysiological analyses was performed as described above but without coverslips. Two days after transfection, cells were divided in order to yield a density of $10^{4}$ cells in a $35-\mathrm{mm}$ dish. These cells were analyzed the following day. Currents were recorded at room temperature $\left(20-22^{\circ} \mathrm{C}\right)$ in the whole-cell patch-clamp configuration using an EPC9 patch-clamp amplifier and Pulse+Pulsefit software (HEKA Elektronik, Lambrecht, Germany). The extracellular solution contained (in mM) $135 \mathrm{NaCl}, 5 \mathrm{KCl}, 2 \mathrm{CaCl}_{2}, 2 \mathrm{MgCl}_{2}, 5$ HEPES, 10 glucose, and 20 sucrose $(\mathrm{pH} 7.4$ with $\mathrm{NaOH}$ ). The intracellular solution contained (in $\mathrm{mM}) 120 \mathrm{~K}$-gluconate, $20 \mathrm{KCl}, 10$ HEPES, 10 EGTA, $2 \mathrm{MgCl}_{2}$ (pH 7.3 with $\mathrm{KOH}$ ), and $1 \mathrm{mM}$ 8-Br-cAMP where indicated. Series resistances ranged from 4-7 $\mathrm{M} \Omega$ and were electronically compensated (60-80\%). Cells were clamped at $-20 \mathrm{mV}$ and hyperpolarized to potentials from $-40 \mathrm{mV}$ to $-120 \mathrm{mV}$ for 5 seconds.
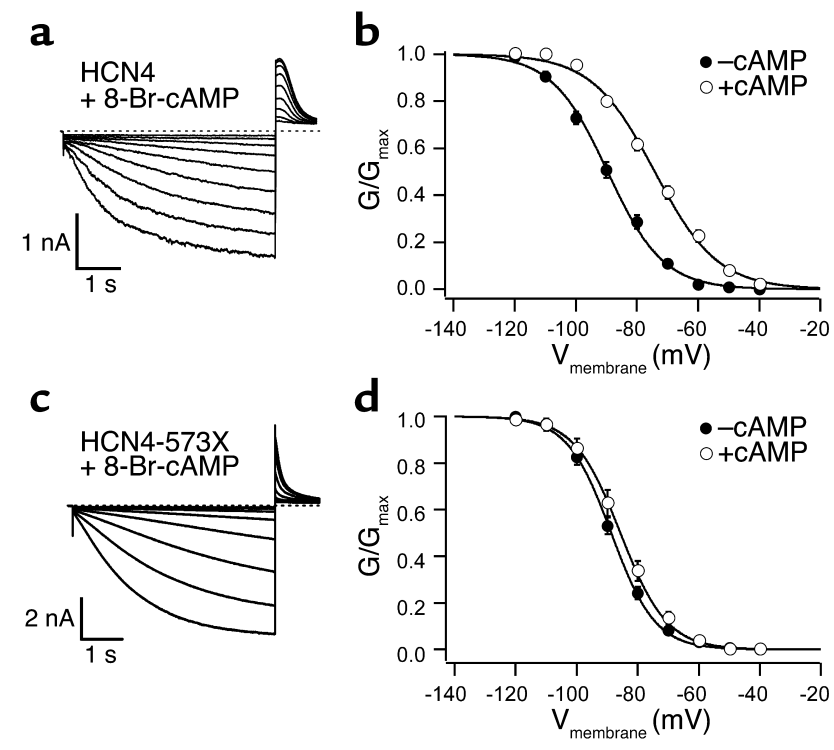

\section{Figure 5}

Functional characterization of homomeric wild-type and HCN4-573X channels in COS-7 cells in the presence of 8-Br-cAMP. Shown are representative current traces of wild-type (a) or HCN4-573X (c) channels elicited by 5 -second-long hyperpolarizing voltage steps from $-40 \mathrm{mV}$ to $-120 \mathrm{mV}$ in the presence of 8-Br-cAMP. Voltage dependence of wild-type (b) and HCN4-573X (d) conductances in the absence (black circles) or presence (white circles) of $1 \mathrm{mM} 8$-Br-cAMP. Absence of error bars indicates errors smaller than the symbol size. 

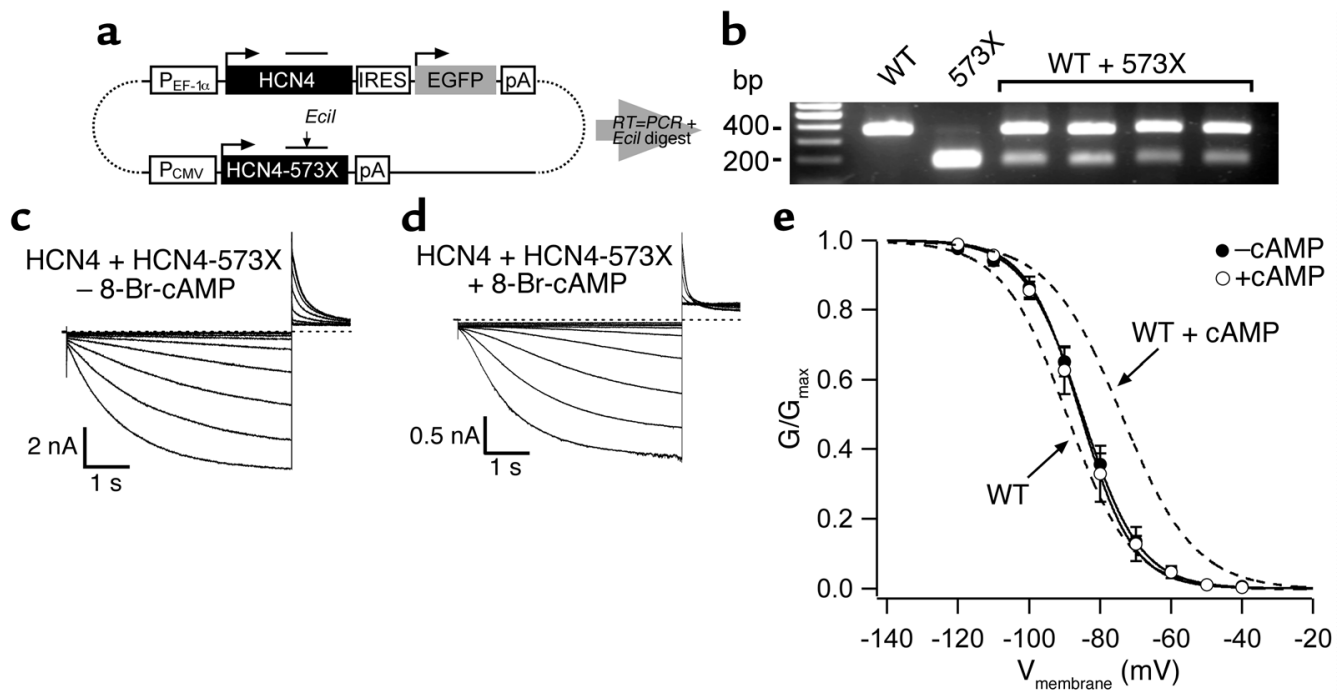

\begin{abstract}
Figure 6
Functional characterization of coexpressed HCN4 and HCN4-573X subunits in COS-7 cells. (a) Schematic representation of the expression construct used for coexpression experiments (not drawn to scale). Wild-type and mutant HCN4 cDNAs are located on the same plasmid (indicated with dotted lines); initiation codons of open reading frames are indicated with horizontal arrows. Structural elements such as promoters ( $\left.\mathrm{P}_{\mathrm{CMV}}, \mathrm{P}_{\mathrm{EF}-1 \alpha}\right)$, IRES, and polyadenylation signals are shown in open boxes. The PCR products amplified from reverse-transcribed total RNA are indicated by a horizontal line above the HCN4 open reading frames. The location of the Ecil restriction site is indicated by a vertical arrow. PA, polyadenylation signal. (b) Agarose gel electrophoresis of Ecil-digested RT-PCR products amplified from total RNA of transiently transfected COS-7 cells. The wild-type fragment has a size of 353 bp and contains no Ecil restriction site. In contrast, the 352-bp-long mutant PCR fragment (573X) is digested by Ecil (see Figure 2c). The resulting two fragments (160 bp and 192 bp) are not separated under the chosen conditions. Shown are representative current traces of coexpressed wild-type and HCN4-573X subunits elicited by hyperpolarizing voltage steps from $-40 \mathrm{mV}$ to $-120 \mathrm{mV}$ in the absence (c) or presence (d) of 8-Br-cAMP. Voltage dependences of heteromeric wild-type/hHCN4-573X (e) conductances in the absence (black circles) or presence (white circles) of 1 mM 8-Br-cAMP are shown. The dashed lines indicate the respective Boltzmann fits of HCN4 (WT) with or without application of 8-Br-cAMP taken from Figure 4. Absence of error bars indicates errors smaller than the symbol size.
\end{abstract}

In addition, 2 -second test pulses up to $-140 \mathrm{mV}$ and 15 -second test pulses up to $-110 \mathrm{mV}$ were applied. The voltage dependence of activation was analyzed by fitting the Boltzmann function $\left(\mathrm{I}-\mathrm{I}_{\min }\right) /\left(\mathrm{I}_{\max }-\mathrm{I}_{\min }\right)$ $=1 /\left(1-\exp \left[\left(\mathrm{V}-\mathrm{V}_{1 / 2}\right) /\right.\right.$ slope factor $\left.]\right)$ to tail currents at $20 \mathrm{mV}$ or $30 \mathrm{mV}$. Time constants of activation were determined by fitting single exponentials to current traces of 15 -second duration. All data are given as means \pm SEM.

\section{Results}

Genomic structure and chromosomal localization of the HCN4 gene

The genomic structure of the HCN4 gene was defined on the basis of several genomic contigs identified by a database search of the NCBI GenBank htgs database. Eight exons, comparable to the structure of the HCN2 gene (17), were identified (Table 2 and Figure 2a). In agreement with the chromosomal in situ hybridization (18), the HCN4 gene was localized to chromosome 15q23-q24.1 near the microsatellite D15S215 (data not shown).

\section{Mutation detection in the HCN4 gene}

We sequenced the HCN4 genes of 10 unrelated index patients. For one index patient, we detected a heterozygous single base-pair deletion (1631delC) in exon 5 of the HCN4 gene (Figure 2b). The deletion gave rise to a new EciI restriction site that was also used for mutation detection (Figure 2c). Restriction digests and sequencing of the DNA from the three available healthy children of this index patient did not show the mutation. Screening of genomic DNA from a sample of the general population (362 chromosomes) failed to detect the mutation, making the presence of a polymorphism unlikely.

The 1631 delC mutation in exon 5 caused a reading frame shift that gives rise to a shortened open reading frame (P544fsX30) and thereby eliminated the HCN4 cNBD. The mutant protein is predicted to be only 572 amino acids long (HCN4-573X) and ends with an HCN4-unrelated sequence of 29 amino acids (residues 544-572) (Figure 2d).

\section{Immunostaining and intracellular trafficking} of wild-type and mutant $\mathrm{HCN} 4$ subunits

To investigate the subcellular localization and intracellular trafficking of HCN4-573X, COS-7 cells were transiently transfected with tagged HCN4 constructs. Subsequent confocal microscopy showed similar immunostaining patterns of comparable intensities with antibodies directed against tagged HCN4 or HCN4-573X proteins. Prominent immunoreactivity was detected in perinuclear regions and at the plasma 
Table 3

Electrophysiological characteristics of wild-type, mutant, and coexpressed HCN4 channels

\begin{tabular}{|c|c|c|c|c|c|}
\hline & \multirow{2}{*}{$\begin{array}{l}\mathrm{HCN} 4 \\
(\mathrm{~A})\end{array}$} & \multirow{2}{*}{$\begin{array}{l}\text { HCN4-573X } \\
\text { (B) }\end{array}$} & \multirow{2}{*}{$\begin{array}{c}\mathrm{HCN} 4 / \mathrm{HCN} 4-573 \mathrm{X} \\
\text { (C) }\end{array}$} & \multicolumn{2}{|c|}{$P$ value } \\
\hline & & & & $A$ versus $B$ & $A$ versus $C$ \\
\hline $\mathrm{V}_{1 / 2}(\mathrm{mV})$ & $-89.6 \pm 1.3^{\mathrm{A}}$ & $-88.7 \pm 1.2^{\mathrm{B}}$ & $-85.0 \pm 1.3^{C}$ & 0.615 & 0.025 \\
\hline Slope factor (mV) & $9.1 \pm 0.3$ & $7.2 \pm 0.4$ & $8.0 \pm 0.5$ & $<0.001$ & 0.089 \\
\hline$\tau_{-110 \mathrm{mV}}(\mathrm{ms})$ & $2377 \pm 546$ & $2028 \pm 433$ & $2032 \pm 266$ & 0.177 & 0.321 \\
\hline $\mathrm{V}_{1 / 2}(\mathrm{mV})$ plus cAMP & $-74.2 \pm 1.2^{\mathrm{A}}$ & $-85.7 \pm 1.9^{\mathrm{B}}$ & $-85.5 \pm 2.5^{C}$ & $<0.001$ & 0.007 \\
\hline Slope factor plus cAMP $(\mathrm{mV})$ & $10.3 \pm 0.2$ & $7.6 \pm 0.2$ & $7.5 \pm 0.4$ & $<0.001$ & $<0.001$ \\
\hline$\tau_{-110 \mathrm{mV}}(\mathrm{ms})$ plus cAMP & $1483 \pm 216$ & $2676 \pm 413$ & $1806 \pm 172$ & $<0.001$ & 0.175 \\
\hline
\end{tabular}

Values are given as means $\pm \mathrm{SEM} . \mathrm{V}_{1 / 2}$ values shown were obtained from 5 -second test pulses. For statistical analysis, a heteroscedastic two-tailed Student's $t$ test was applied. ${ }^{A} P<0.001 .{ }^{B} P=0.206 .{ }^{C} P=0.879$.

membrane of COS-7 cells (Figure 3, a and b). The HCN4 and HCN4-573X immunostaining patterns completely overlapped when wild-type and mutant $\mathrm{HCN} 4$ subunits were coexpressed (Figure 3, c to e). The observed overlapping immunoreactivity in the cell membranes (Figure 3f) suggested that the subunits were colocalized. Thus, the immunohistochemical results indicated that intracellular trafficking and processing of HCN4 and HCN4-573X proteins were similar.

\section{Functional expression of wild-type} and mutant HCN4 channels

The whole-cell configuration of the patch-clamp technique was used to functionally characterize HCN4-573X channels transiently expressed in COS-7 cells. Upon hyperpolarizing steps in membrane potential $(-40 \mathrm{mV}$ to $-120 \mathrm{mV}$ for 5 seconds), HCN4-573X channels mediated, like wild-type HCN4 channels, hyperpolarizationactivated currents with typical $I_{f}$ features $(17,18)$ (Figure 4). Activation kinetics of HCN4 and HCN4-573X currents did not differ significantly and were reasonably fit with a single exponential function $\left(\tau_{-110 \mathrm{mV}}=\right.$ $2377 \pm 546 \mathrm{~ms}[n=11]$ versus $2028 \pm 433 \mathrm{~ms}[n=12])$ (Table 3). In agreement with a previous report (17), HCN4 channels did not reach steady state during the 5 -second activation protocol (Figure 4a). However, the relative open probabilities of HCN4 and HCN4-573X channels taken from instantaneous outward tail currents were reliably described by Boltzmann functions. The midpoint potentials $\left(\mathrm{V}_{1 / 2}\right)$ of HCN4 and HCN4$573 \mathrm{X}$ channels were very similar $(\mathrm{HCN} 4,-89.6 \pm 1.3$ $\mathrm{mV}[n=11] ; \mathrm{HCN} 4-573 \mathrm{X},-88.7 \pm 1.2 \mathrm{mV}[n=11]$; $P=0.61)$ for the 5 -second hyperpolarization protocol (Figure 4 and Table 3). Because of the slow activation kinetics of HCN channels, $V_{1 / 2}$ strongly depended on the test-pulse duration. Two-second test pulses yielded $\mathrm{V}_{1 / 2}$ values of $-107.0 \pm 2.0 \mathrm{mV}(n=7)$ for $\mathrm{HCN} 4$ versus $-110.2 \pm 1.2 \mathrm{mV}(n=10)$ for $\operatorname{HCN} 4-573 \mathrm{X}(P=0.21)$. Test pulses of 15 seconds resulted in $V_{1 / 2}$ values of $-70.2 \pm 2.2 \mathrm{mV}(n=7)$ for $\mathrm{HCN} 4$ versus $-76.0 \pm 0.8 \mathrm{mV}$ $(n=8)$ for HCN4-573X $(P=0.043)$ (Figure 4). Thus, for long activation protocols, the midpoint of activation of HCN4-573X channels was approximately $6 \mathrm{mV}$ more negative than of wild-type channels.
The deactivation kinetics of HCN4 channels displayed a sigmoidal time course. In contrast, the deactivation kinetics of HCN4-573X channels were exponential and therefore more closely resembled those of HCN1 and HCN2 than of HCN4 channels.

A hallmark of HCN channels is their regulation by cyclic nucleotides. Since the HCN4-573X channels lacked the C-terminal $\mathrm{cNBD}$, we investigated the effect of increased cAMP levels on HCN4-mediated currents. We used the nonhydrolyzable cAMP analog 8-Br-cAMP to assess the sensitivity of HCN4 channels to intracellular cAMP levels and compared cAMPtreated cells with cells under control conditions. Upon intracellular application of 8-Br-cAMP, the midpoint of activation of $\mathrm{HCN} 4$ currents was shifted approximately $15 \mathrm{mV}$ toward more depolarized membrane potentials $\left(\mathrm{V}_{1 / 2}=-74.2 \pm 1.2 \mathrm{mV}[n=7]\right)$ (Figure $5, \mathrm{~b}$ and $\mathrm{c}$, and Table 3 ). In contrast, the voltage dependence of HCN4-573X current activation was not significantly altered by 8 -Br-cAMP application $\left(\mathrm{V}_{1 / 2}\right.$ plus 8 - $\mathrm{Br}$-cAMP $=-85.7 \pm 1.9 \mathrm{mV}[n=8]$ ) (Figure 5, c and d, and Table 3).

The in vitro expression results suggested that the index patient may express mutant $I_{f}$ channels that have an altered sensitivity to changes in intracellular cAMP concentrations. Since the 1631delC mutation in the index patient's $\mathrm{HCN} 4$ gene was heterozygous, we also simulated the heterozygous condition by coexpressing HCN4 and HCN4-573X subunits. In order to obtain reliable coexpression of wild-type and mutant subunits, a bicistronic expression plasmid was generated that harbored full-length wild-type and mutant HCN4 (Figure 6a). In addition, the EGFP expression was coupled to wild-type HCN4 expression through an IRES element that enabled the selection of transfected cells (see the Methods). Since the expressions of HCN4IRES-EGFP and HCN4-573X were driven by different promoters (Figure 6a), we investigated the relative transcript amounts in transiently transfected COS-7 cells by RT-PCR. We made use of the EciI restriction site, which is only present in mutant transcripts, to discriminate between wild-type and mutant PCR products (see Figure 2c). The relative molecular ratio of wild-type/mutant transcripts was 2:1.05 $\pm 0.2(n=4)$ 
(Figure 6b). This finding indicates a higher efficiency of the EF-1 $\alpha$ promoter controlling the wild-type HCN4-IRES-EGFP expression. COS-7 cells coexpressing wild-type HCN4 and HCN4-573X subunits mediated currents with activation parameters comparable to homomeric wild-type HCN4 and HCN4-573X channels $\left(\mathrm{V}_{1 / 2}=-85.0 \pm 1.3 \mathrm{mV}[n=7]\right)$ (Figure 6, $\mathrm{c}$ and $\mathrm{e}$, and Table 3). Surprisingly, tail currents decayed with an exponential time course similar to HCN4-573X tail currents (Figure 6c). Furthermore, channel exposition to 8-Br-cAMP did not lead to a depolarizing shift in the current-voltage relationship or to a change in activation kinetics $\left(\mathrm{V}_{1 / 2}=-85.5 \pm 2.5 \mathrm{mV}[n=5]\right.$ ) (Figure 6, $\mathrm{d}$ and $\mathrm{e}$, and Table 3 ). These results suggested that coexpression of HCN4 and HCN4-573X subunits also yields cAMP-insensitive channels with altered tail current properties. It is therefore likely that the del1653C mutation acts in a dominant-negative manner.

\section{Discussion}

Spontaneous electrical activity of the mammalian heart and the adaptation of heart rate by the autonomic tone are physiological key features. Recent twin studies have provided evidence for a genetic determination of heart rate and baroreflex function $(8,9,27)$. In the Framingham Heart Study, an analysis of siblings showed that heritable factors account for a substantial proportion of the variance in heart rate and heart rate variability (28). Moreover, several reports of inherited SND with autosomal dominant inheritance were published and indicated the presence of a monogenic disorder in some forms of idiopathic disease.

$I_{f}$ channels were identified to play a role in cardiac pacemaking in the slow mo variant of zebrafish that is characterized by complete absence of the fast $I_{f}$ component of hyperpolarization-activated pacemaker currents and a twofold reduction in heart rate during embryonic and adult stages $(29,30)$. In humans, a contribution of $I_{f}$ channels in the pacemaking activity of the SAN has been discussed controversially $(31,32)$. It is likely that slow and/or fast hyperpolarization-activated pacemaker currents represent key determinants of spontaneous diastolic depolarization in the SAN. The fast component of $I_{f}$ is most likely HCN2-dependent and the slow component HCN4-dependent (17, 18). We previously investigated the $H C N 2$ gene as a candidate for SND. However, we did not find HCN2 gene mutations in a study population with idiopathic SND (24). The present finding of a mutation in the $H C N 4$ gene of one index patient suggests that a genetically determined alteration of cardiac pacemaker channel activity causes SND.

The identified mutation eliminates the functionally important $\mathrm{CNBD}$ in the derived HCN4 subunit sequence because of a truncation of the C-terminus. Two recent reports showed a role for the $\mathrm{C}$-terminus of $\mathrm{HCN} 1$ and $\mathrm{HCN} 2$ channels in the control of voltage and cAMP-mediated channel gating $(33,34)$. The results are compatible with our findings in electrophysiological recordings on transfected COS-7 cells, in which C-terminally truncated HCN4 channels showed regular intracellular trafficking and mediated $I_{f}$-like currents but were unresponsive to increases in the intracellular cAMP concentration. Since the patient's HCN4 mutation was found in a heterozygous state, we also coexpressed wild-type and mutant HCN4 subunits. Like homomeric HCN4-573X channels, coexpressed HCN4-573X/HCN4 channels did not respond to a rise in intracellular cAMP with a shift in their currentvoltage relationship. In addition, the exponential time course of channel deactivation resembled that of HCN4-573X channels. Taken together, these data suggested a dominant-negative effect of mutant $\mathrm{HCN} 4$ on wild-type channel function.

Despite intensive clinical examination of the index patient, there was no obvious cause for SND, nor was there any other disease-associated condition. Thus, the patient with the HCN4 gene mutation was classified as having idiopathic disease. Since the patient's family history revealed no further information regarding SND, we were not able to follow the genetic transmission of the mutation to other family members and thus cannot provide further genetic evidence that the mutation is disease causing. The index patient's phenotype was characterized by a remarkably low heart rate resulting from sinus bradycardia (Figure 1a), intermittent episodes of atrial fibrillation (Figure 1b), and chronotropic incompetence during exercise ECG even under maximum work load (Figure 1d). After pacemaker implantation, retrospective evaluation of the atrial rates over a period of several months was possible. In the P-wave histograms obtained after pacemaker interrogation, consistently low atrial rates were noted (Figure 1c).

Our in vitro expression data strongly support the notion that the patient suffered from SND due to the expression of mutant HCN4 channels, which are insensitive to intracellular cAMP and have altered activation and deactivation properties. Chronotropic incompetence is likely to occur when $I_{f}$ channels cannot respond to increases in intracellular cAMP concentrations during $\beta$-adrenergic stimulation. The sinus bradycardia observed under resting conditions, on the other hand, might be explained by the altered deactivation kinetics of HCN4-573X channels; because of the extremely slow activation kinetics of HCN4 channels, cumulative current activation during repetitive heart beat cycles, as demonstrated for KCNQ1 channels (35), might occur. Faster closure (deactivation) of $I_{f}$ channels during sinus nodal action potentials therefore possibly decreases channel availability in the subsequent cycle, thereby slowing the heart rate. Detailed modeling studies would be necessary to underscore this hypothesis. Considering the broad phenotypic spectrum of arrhythmias associated with familial SND $(2,4-7)$, it is likely that additional genes and molecular mechanisms for this "idiopathic" disease will be defined. 
Note added in proof. Further experimental support for a role of HCN channels in cardiac pacing was recently provided by Fikret et al. (36) who showed that these channels are critical for spontaneous beating of rat neonatal cardiomyocytes.

\section{Acknowledgments}

We gratefully acknowledge the excellent technical work of Ellen Schulze-Bahr, Marielies Hesse, Kathrin Sauter, Andrea Zaisser, and Anne Schneider-Darlison. We are indebted to the patients and individuals that participated in the present study, which was in accordance with the ethical standards in the revised version of the Declaration of Helsinki and with ethical recommendations given by the local ethics committee. This work was partially supported by grants from the Innovative Medizinische Forschung of the University of Muenster (IMF; SC 2_1_99_25), from the Deutsche Forschungsgemeinschaft (PF1625/1-1), and from Fondation Leducq (Paris, France).

1. Lamas, G.A., et al. 2000. The mode selection trial (MOST) in sinus node dysfunction: design, rationale, and baseline characteristics of the first 1000 patients. Am. Heart J. 140:541-551.

2. Sarachek, N.S., and Leonard, J.L. 1972. Familial heart block and sinus bradycardia. Classification and natural history. Am. J. Cardiol. 29:451-458.

3. Spellberg, R.D. 1971. Familial sinus node disease. Chest. 60:246-251.

4. Lehmann, H., and Klein, U.E. 1978. Familial sinus node dysfunction with autosomal dominant inheritance. Br. Heart J. 40:1314-1316.

5. Mackintosh, A.F., and Chamberlain, D.A. 1979. Sinus node disease affecting both parents and both children. Eur. J. Cardiol. 10:117-122.

6. Bertram, H., Paul, T., Beyer, F., and Kallfelz, H.C. 1996. Familial idiopathic atrial fibrillation with bradyarrhythmia. Eur. J. Pediatr. 155:7-10.

7. Brugada, R., et al. 1997. Identification of a genetic locus for familial atrial fibrillation. N. Engl. J. Med. 336:905-911.

8. Russell, M.W., Law, I., Sholinsky, P., and Fabsitz, R.R. 1998. Heritability of ECG measurements in adult male twins. J. Electrocardiol. 30(Suppl.):64-68.

9. Van Hulle, C.A., Corley, R., Zahn-Waxler, C., Kagan, J., and Hewitt, J.K. 2000. An exploration of the genetic and environmental etiology of heart rate in infancy and middle childhood. Twin Res. 3:259-265.

10. Schram, G., Pourrier, M., Melnyk, P., and Nattel, S. 2002. Differential distribution of cardiac ion channel expression as a basis for regional specialization in electrical function. Circ. Res. 90:939-950.

11. Brown, H.F., DiFrancesco, D., and Noble, S.J. 1979. How does adrenaline accelerate the heart? Nature. 280:235-236.

12. DiFrancesco, D., Ducouret, P., and Robinson, R.B. 1989. Muscarinic modulation of cardiac rate at low acetylcholine concentrations. Science. 243:669-671.

13. Kaupp, U.B., and Seifert, R. 2001. Molecular diversity of pacemaker ion channels. Annu. Rev. Physiol. 63:235-257.
14. Ludwig, A., Zong, X., Hofmann, F., and Biel, M. 1999. Structure and function of cardiac pacemaker channels. Cell Physiol. Biochem. 9:179-186. 15. Ludwig, A., Zong, X., Jeglitsch, M., Hofmann, F., and Biel, M. 1998. A family of hyperpolarization-activated mammalian cation channels. Nature. 393:587-591.

16. Santoro, B., et al. 1998. Identification of a gene encoding a hyperpolarization-activated pacemaker channel of brain. Cell. 93:717-729.

17. Ludwig, A., et al. 1999. Two pacemaker channels from human heart with profoundly different activation kinetics. EMBO J. 18:2323-2329.

18. Seifert, R., et al. 1999. Molecular characterization of a slowly gating human hyperpolarization-activated channel predominantly expressed in thalamus, heart, and testis. Proc. Natl. Acad. Sci. U. S. A. 96:9391-9396.

19. Vaccari, T., et al. 1999. The human gene coding for HCN2, a pacemaker channel of the heart. Biochim. Biophys. Acta. 1446:419-425.

20. Shi, W., et al. 1999. Distribution and prevalence of hyperpolarizationactivated cation channel $(\mathrm{HCN})$ mRNA expression in cardiac tissues. Circ. Res. 85:e1-e6.

21. Moosmang, S., et al. 2001. Cellular expression and functional characterization of four hyperpolarization-activated pacemaker channels in cardiac and neuronal tissues. Eur. J. Biochem. 268:1646-1652.

22. Gauss, R., Seifert, R., and Kaupp, U.B. 1998. Molecular identification of a hyperpolarization-activated channel in sea urchin sperm. Nature. 393:583-587.

23. Ishii, T.M., Takano, M., Xie, L.H., Noma, A., and Ohmori, H. 1999. Molecular characterization of the hyperpolarization-activated cation channel in rabbit heart sinoatrial node. J. Biol. Chem. 274:12835-12839.

24. Schulze-Bahr, E., et al. 1999. The cardiac pacemaker channel gene, HCN2, is not linked with congenital sinus node dysfunction and AV-conduction block. Am. J. Hum. Genet. 65(Suppl. S):2787. (Abstr.)

25. Farazdaghi, G.R., and Wohlfart, B. 2001. Reference values for the physical work capacity on a bicycle ergometer for women between 20 and 80 years of age. Clin. Physiol. 21:682-687.

26. Schulze-Bahr, E., et al. 1995. Molecular analysis at the Harvey Ras-1 gene in patients with long QT syndrome. J. Mol. Med. 73:565-569.

27. Tank, J., et al. 2001. Genetic influences on baroreflex function in normal twins. Hypertension. 37:907-910.

28. Singh, J.P., et al. 1999. Heritability of heart rate variability: the Framingham Heart Study. Circulation. 99:2251-2254.

29. Baker, K., Warren, K.S., Yellen, G., and Fishman, M.C. 1997. Defective "pacemaker" current (Ih) in a zebrafish mutant with a slow heart rate. Proc. Natl. Acad. Sci. U. S. A. 94:4554-4559.

30. Warren, K.S., Baker, K., and Fishman, M.C. 2001. The slo mo mutation reduces pacemaker current and heart rate in adult zebrafish. Am. J. Physiol. Heart Circ. Physiol. 281:H1711-H1719.

31. Irisawa, H., Brown, H.F., and Giles, W. 1993. Cardiac pacemaking in the sinoatrial node. Physiol. Rev. 73:197-227.

32. Vassalle, M., Yu, H., and Cohen, I.S. 1995. The pacemaker current in cardiac Purkinje myocytes. J. Gen. Physiol. 106:559-578.

33. Wainger, B.J., DeGennaro, M., Santoro, B., Siegelbaum, S.A., and Tibbs, G.R. 2001. Molecular mechanism of cAMP modulation of HCN pacemaker channels. Nature. 411:805-810.

34. Viscomi, C., et al. 2001. C terminus-mediated control of voltage and cAMP gating of hyperpolarization-activated cyclic nucleotide-gated channels. J. Biol. Chem. 276:29930-29934.

35. Romey, G., et al. 1997. Molecular mechanism and functional significance of the MinK control of the KvLQT1 channel activity. J. Biol. Chem. 272:16713-16716.

36. Er, F., et al. 2003. Dominant-negative suppression of HCN channels markedly reduces the native pacemaker current $I(f)$ and undermines spontaneous beating of neonatal cardiomyocytes. Circulation. 107:485-489. 